\title{
PELVIC THROMBOSIS
}

\author{
By J. Stallworthy, F.R.C.S., F.R.C.O.G. \\ Radcliffe Infirmary, Oxford
}

' By nature's kindly disposition most questions which it is beyond man's power to answer do not occur to him at all.'-Santayana.

Wherever the blessings of modern surgery and obstetrics were available there can have been few families 25 years ago with no experience among their circle of relatives and friends of sudden death from embolism, or of prolonged illness from venous thrombosis and white leg. A quarter of a century has changed this tragic picture almost beyond recognition. Death remains the greatest and final threat to life, but the number of patients departing through the portal marked 'Embolism' has steadily decreased. The Registrar-General recorded as due to pulmonary embolism, sudden death, and phlegmasia dolens, I,434 puerperal deaths in England and Wales for the six years ending 1927, an average of 239 a year. This was the equivalent of 0.3 deaths per 1,000 live births at a time when the total mortality was 3.9 per 1,000 . In 1947 , however, there were 56 deaths from pulmonary embolism; in 1948,36 ; and in 1949 only 26 , a reduction of over half in these three years. Viewed from a different angle, it should now be possible for 20,000 or more consecutive unselected deliveries to take place without a maternal death from pulmonary embolism.

In a series of over 15,000 consecutive deliveries conducted in the Area Department of Obstetrics, United Oxford Hospitals, since 1949 there was one death from pulmonary embolism. This was in spite of a high incidence of abnormal and emergency cases requiring instrumental or surgical intervention.

In the same way the surgical risks have decreased. Figures published from the Mayo Clinic by Barker and his associates (1940-4I) recorded an incidence of pulmonary embolism of 0.5 per cent. in 172,888 operations of all types and it was fatal in nearly half, an incidence of 0.2 per cent. The same author in 1944 stated that the incidence of thrombosis after abdominal hysterectomy was 4 per cent. and that the risk was increased tenfold when there was a history of previous thrombus or embolism. This high association of thrombosis and gynaecological operations has been recorded by other writers, including Oschner and De Bakey $\stackrel{\vec{D}}{\circ}$ (1939), and Campbell and Blahey (1950), who $\vec{\omega}$ reported venous thrombosis in 1.33 per cent., embolism in 1.08 per cent., and fatal embolism in 8 0.25 per cent. of all types of hysterectomies performed over a series of 20 years at the Montreal $\omega_{0}$ General Hospital. The risk of sub-total hysterec- $\omega$ tomy was twice that of the total, or vaginal opera- of tions. Figures from the Lahey Clinic were given by $\dot{\sigma}^{\prime}$ Evans and Dee (195I). There were 238 instances of thrombosis and embolism in 56,000 major 을 operations, or 0.4 per cent., but of these there were $\overrightarrow{ }$ 54 sudden deaths from pulmonary embolism in patients who had, it is stated, given neither signs nor symptoms of thrombosis, an incidence of $\overrightarrow{0}$ approximately o.I per cent. This was in spite \&े the fact that precautions to reduce the risk of thrombosis had been taken in the surgical care of the patients in this series.

Murray (1950) contrasted 5 per cent. of thrombosis following gynaecological operations and prostectomy with less than 0.5 per cent. in operations of all kinds, but in his Hunterian lecture at the Royal College of Surgeons, Dew (1953) recorded only two fatal emboli in a series of 530 prostatectomies.

Haines (r95I) recorded 55 cases of fatal pulmonary emboli in a total of 312 deaths from all causes occurring in over 30,000 gynaecological operations performed during 20 years at Chelsea $\delta$ Hospital for Women, London. This is an incidence of . 8 per cent. He noted, however, that operative $ᄋ$ and post-operative deaths from all causes, in- $D$ cluding emboli, had gradually decreased.

In the last $I 1, \infty 00$ consecutive gynaecological $\bar{N}$ operations performed in the Area Department at Oxford there were five deaths from embolism, an incidence of 0.04 per cent.

From the above reports it is apparent that the risk of pulmonary embolism is less in obstetrics than gynaecology, is steadily decreasing in both, but is a major hazard in relation to the reduced ? mortality from all causes.

Before endeavouring to assess the possible reasons for this improvement certain fundamental 
facts will be reviewed concerning the pathology of post-operative and obstetric thrombosis.

\section{Pathology}

De Takats (1940) described the normally circulating blood as having an axial corpuscular stream with a marginal flow of plasma containing an occasional leucocyte. When the circulation is slowed the axial stream becomes narrowed and the rate of marginal flow greatly reduced. Moreover, the plasma contains increasing numbers of leucocytes and platelets closely applied to the vessel wall. The retardation of circulation does not usually of itself lead to thrombosis, and one of the factors preventing this is the presence of heparin (the blood's own anticoagulant), which is found in greatest concentrations in the neighbourhood of small blood vessels. The platelets may, however, agglutinate and disintegrate with the formation of thromboplastin. This substance in the presence of ionized calcium salt activates prothrombin (which normally is produced continuously by the liver, enters the circulation and rapidly disappears from the circulating blood) to form thrombin. Acting as an enzyme on fibrinogen, this liberates the. insoluble hydrogel fibrin which forms the framework on which clot develops. This whole process is initiated once thromboplastin is formed in quantity by platelets degenerating, or tissue injury by trauma, infection, or anoxia. If clotting is arrested in its early stages, the initial fibrin clot may disappear as the result of phagocytic activity.

When a thrombus develops in this way a red coagulation clot may be superadded to the initial white thrombus formed round the disintegrating platelets and adherent to the vessel wall. The red clot may enlarge extremely rapidly. It extends along the vein to the point of its junction with another vein where blood flowing in the undamaged vessel tends to arrest the process. The clot may organize and the lumen again canalize; it may extend into the proximal vessel, or it may fragment to produce emboli which are transported to the heart by the more rapidly flowing stream in the undamaged vein. A white thrombus is firmly adherent to the vessel wall and emboli are less likely to form from it than from the red variety. This would explain the findings of Evans and Dee (195I) that fatal pulmonary emboli were more frequent after operation in those patients who had no demonstrable evidence of thrombosis prior to the terminal catastrophe than in those with a demonstrable peripheral thrombosis. When infection occurs, not only are there usually local signs and symptoms, but if the vessel wall is damaged the resulting white thrombus tends to be adherent to the intima and fragmentation and emboli are less likely to occur.
The experience of the Lahey Clinic, as recorded by Evans and Dee, that 54 sudden deaths due to pulmonary emboli were not preceded by clinical evidence of peripheral thrombosis, can, however, be explained in another way. At autopsy, when death has occurred from pulmonary embolism, thrombosis of veins in the lower limb is frequently not demonstrated. Dew (1953) states that it is present in only 50 per cent., whereas in 90 per cent. vessels in the lung bases contain thrombi. He argues from this and other evidence that primary pulmonary thrombosis is more common than is usually thought. If this is so, many deaths from so-called emboli are, in fact, due to primary thrombosis in the pulmonary vessels. This is more than an academic point, for, if established, it would justify on general principles the reluctance of British surgeons to follow the lead of some of their American colleagues in performing peripheral venous ligation proximal to sites of thrombosis as a means of preventing pulmonary embolism. It would also. support the need for prophylactic therapy as. discussed later.

It should be recorded, however, that Haines (195I) expresses the view that peripheral thrombosis can be missed at autopsy because the examination is not sufficiently extensive. He described a technique for displaying the muscles of the calf and stated that, whereas the gastrocnemius. is seldom affected, the soleus muscle and deep. veins usually contain ante-mortem thrombi. They may even be found in the plantar veins of the foot. $\mathrm{He}$ believes that pulmonary emboli in obstetrics and gynaecology are associated with primary thromboses in these sites.

At this stage it should be mentioned that in 1940 Bergquist postulated a prethrombotic state which he believed was present for approximately 12 hours. before clotting occurred. During this time coagulation tests recorded falling values and he accepted three minutes as the danger level. Cummine and Lyon (1948), working in Sydney, modified the technique to use capillary tubes and blood from the pricked finger in place of venepuncture and decided on four minutes as the danger level. They described a substance called fibrinogen B, which they believed was an intermediate product between fibrinogen and fibrin, which if present in excess with a reduced coagulation time made thrombosis inevitable unless adequate therapy was immediately instituted. Ryan (195I), of Toronto, working on similar lines, found that fibrinogen $B$ was present in greatest amount usually seven to nine days after operation, a fact which would explain the relatively high incidence of thrombosis and emboli at this stage of convalescence.

Oschner and De Bakey (1940) and Homans (1943) described two types of venous thrombosis, 
thrombo-phlebitis and phlebo-thrombosis. In the former the vessel wall is damaged by infection and when clotting occurs the thrombus remains attached to the intima. In the latter there is no evidence of inflammation and the clot formed in the lumen of the vessel is unattached and embolism is therefore more likely to occur. Factors responsible are stasis, trauma and toxins (as, for example, from degenerating tissue in malignant diseases, or even in benign tumours, such as fibroids). It is not uncommon at operation to find thrombi in the uterine vessel, where there is neither history nor local evidence of infection. This classification into thrombo-phlebitis and phlebo-thrombosis probably over-simplifies the true position and it is possible that in some cases at least the two conditions are but differing stages of the one disease. If the concept of primary pulmonary thrombosis as opposed to pulmonary embolism becomes accepted as the more usual explanation of the acute pulmonary lesion, the role of so-called phlebothrombosis in the aetiology of pulmonary embolism will be less important than has hitherto been believed.

\section{Clinical Picture}

Intravenous clotting is more common in the aged than in the young; in patients with cardiac disease and varicose veins than in those with a healthy cardio-vascular system; in the anaemic and debilitated, and in those with malignant disease. In fact, femoral thrombosis is on rare occasions the first indication of unsuspected abdominal cancer. When venous thrombosis has occurred in the ante-natal period or before operation, the danger of puerperal or post-operative recurrence is greatly increased.

The clinical picture may be obvious or confusing. In the former there is pain and swelling in the lower limb (usually the left) which may involve only the calf or the thigh as well, according to the site of the thrombus. Pain may be very severe, of sudden onset and associated with an initial pallor of the limb, followed in a few hours by blueing and oedema. Most patients with a deep femoral thrombosis have pain over the region of the femoral ring, but occasionally pain is confined to the calf in spite of the fact that oedema extends from the inguinal ligament to the foot. De Bakey, Burch and Oschner (1939) drew attention to the importance of vascular spasm in producing these signs and symptoms. The reflex initiated in the damaged segment of vein is referred through the sympathetic system to the remainder of the vascular tree, arterial and venous, of the affected limb. Evidence in support of this view is provided by the dramatic response to local infiltration of the autonomic system if this is performed in the early stages of the attack. Pain is relieved, the limb becomes warm and pink and gross oedema resultant on endothelial damage by prolonged anoxia is avoided. In the same way the obvious classical picture of pulmonary embolism (or primary pulmonary thrombosis) is not likely to be mistaken. The anxious facies, the associated shock and dyspnoea, the story of the wanted bed-pan, and the sudden terrible thoracic pain, are all so typical in the puerperal or post-operative patient. The same catastrophe can happen in the ward or the anaesthetic room before operation. Unfortunately, in both the above types of case the condition of the patient is grave and it may be too late for the most vigorous treatment to save life. There is considerable doubt, however, whether even in these socalled unheralded cases it is true to say that there were no premontary signs for those with eyes to see them. For example, the pulse chart usually shows that the post-operative or puerperal pulse has been elevated for some days above the level one would expect with a normal case. There may be the odd spiking of temperature, even as little as $99^{\circ}$. Not uncommonly the patient has not felt well for 24 hours or so with either nausea or anorexia. In such cases careful questioning and examination will sometimes produce evidence of an aching in the calf, a resentment to dorsiflexion of the foot and to actual tenderness over the calf muscles.

The confusing cases deserve more attention for prompt diagnosis leading to immediate effective treatment should in all cases restore the sword of Damocles to its scabbard.

The confusing pulmonary case is often misdiagnosed as one of consolidation, collapse or broncho-pneumonia, usually because the clinician is not 'thrombosis conscious.' If the patient is one of those already described as being particularly liable to develop intra-vascular clotting, there is less reason for this error. The increased pulse rate or recurrent elevation of temperature should arouse suspicion before local signs develop and result in the coagulation time being estimated. If this is decreased to three minutes or less, anticoagulant therapy should at once be commenced. It should be remembered that a chest X-ray will distinguish between a broncho-pneumonia and infarction due to an embolus. The former reveals a shadow extending to the hilus and the latter a triangular opacity with its base on the pleura.

Mention should be made of a fortunately rare type of pelvic vein thrombosis seen in the puerperium and post-abortal state, in which there is infection with an anaerobic streptococcus. This produces a suppurative thrombo-phlebitis in which recurrent small infected emboli are released into the circulation. Each shower of emboli is associated with a rigor and may be followed by pyaemic abscesses throughout the body. The im- 
portance of anaerobic blood cultures in this type of case is obvious.

\section{Treatment}

Prophylaxis. An important factor in reducing the incidence of thrombosis and embolism in obstetrics and gynaecology in recent years is undoubtedly the increased attention given to adequate ante-natal care and to the pre-operative preparation of patients. The adequate treatment of anaemia and focal sepsis are obvious examples. Attention to carbohydrate tolerance as estimated by blood sugar curves is stressed by Campbell and Blahey (1950) as being of particular importance in the gynaecological patient. Also important is the recognition and treatment of sub-thyroidism. In fact, Best and Taylor (1945) advised giving thyroid extract in the pre-operative phase to increase the basal metabolic rate and pulse rate. Improved techniques to reduce the incidence of sepsis and skilled anaesthesia to aid the gentle handling of tissues all play their part, while in obstetrics the avoidance of dehydration in labour, the reduction of blood loss and timely intervention in prolonged labour are equally essential. The position of the patient on the delivery or operating table is important. Flexion of the thighs, pressure on the calves and the extreme Trendelenberg position are all important in causing stasis and anoxia.

In the immediate post-operative and postpartum phase both passive and active movement are invaluable and the skilled and conscientious physiotherapist is a most important member of the team in reducing the incidence of intra-vascular clotting. The avoidance of tight constricting abdominal bandages and the early commencement of deep breathing are to be commended.

Once signs or symptoms of clotting occur anticoagulant therapy should at once be instituted with or without specific chemotherapy.

Anticoagulant Therapy. An initial dose of 10,000 units of heparin (100 mg.) should be given intravenously and a subsequent dose adjusted according to the clotting time which should be maintained at double the normal figure. If over-dosage occurs, spontaneous bleeding may commence at the operation site, in the kidneys, in the brain, in the skin, or elsewhere, and protamine sulphate (100 mg.) should be given intravenously and repeated, if necessary; I $\mathrm{mg}$. neutralizes approximately I $\mathrm{mg}$. of heparin. The effect of intravenous heparin is often dramatic. The patient with a misdiagnosed pulmonary embolism which has been treated without relief as a broncho-pneumonia with antibiotic drugs will undergo dramatic clinical improvement once heparin is given. The severe pain of a femoral thrombosis will rapidly disappear in many cases and the question of a sympathetic block to break the reflex arc will no longer need consideration. Once heparin has been given for its rapid effect it is usually easier for the patient and more convenient for the doctor to avoid further intravenous therapy and continue treatment by one of the synthetic oral preparations, such as dicoumarol, tromexan and, more recently, dindevan or marcoumar. Early experience with dicoumarol was not entirely happy and as its effect lasts longer than heparin and the newer preparations these are used instead of it in many clinics. Dindevan is given by mouth in doses of 50 to I00 mg. twice daily, the dose being adjusted according to the prothrombin time, which should be maintained at from ro to 20 per cent. of the normal figure. Once the local signs have regressed, active movement is encouraged and the patient should get out of bed, even though the anticoagulant drugs are continued for 10 to 14 days in all.

\section{Summary}

I. There has been a steady fall in the incidence of fatal pulmonary embolism in both obstetrics and gynaecology.

2. The risk is greater in gynaecology than in obstetrics.

3. Age, cardiac pathology, anaemia, stasis, sepsis, toxins (from degenerating tumours) and local trauma all predispose to thrombosis.

4. A high standard of obstetrical and gynaecological care (ante-partum, intra-partum, postpartum; pre-operative, operative and post-operative) can reduce the risk of embolism to a minimum and the fatal case should seldom be seen.

\section{BIBLIOGRAPHY}

BARKER, N. W., et al. (1940), Proc. Mayo Clinic, 15, 769.

BARKER, N. W., et al. (I94I), Ibid., I6, I, 17, 33.

BEST, C. H., and TAYLOR, N. B. (r945), 'The Physiological Basis of Medical Practice,' 4th edition, Baltimore.

CAMPBELL, A. D., and BLAHEY, P. R. (r950), 'Modern Trends in Obstetrics and Gynaecology,' p. 627, Butterworth.

CUMMINE, H., and LYONS, R. N. (1948), Brit. F. Surg., 35, 338.

DE BAKEY, M. E., BURCH, C. E., and OSCHNER, A. (1939), Proc. Soc. exp. Boil., N.Y., 41, 585.

DE TAKATS, G., et al. (1940), F.A.M.A., I14, 1415.

DEW, H. (1953), Annals of Roy. Col. Surg. of Eng., 13, I.

EVANS, J. A., and DEE, J. F. (195I), 'Surgical Practice of the Lahey Clinic,' p. 926, Saunders.

HAINES, M. (1951), Amer. F. Obs. E Gynec., 6ra, 260.

HOMANS, J. (1943), New Eng. Med. F., 211 , 993.

HOMANS, J. (1944), Ibid.,, 231, 5 r.

HOMANS, J. (1946), Ibid., 235, I63, 193.

HOMANS, J. (1946), Ibid., 235, 249.

MURRAY, G. (1950), 'British Surgical Practice,' p. 234, Butterworth.

OSCHNER, A., and DE BAKEY, M. (1939), Sth. Surg., 8, 269.

OSCHNER, A., and DE BAKEY, M. (1940), Arch. Surg., 40, 268. RYAN, E. A. (r95I), Canad. med. Ass. F., 84, 237. 